be antibody limited, might be beneficial ${ }^{12}$. The persistent immune responses observed by Hammarlund et al. suggest that side effects of vaccination, such as eczema vaccinatum ${ }^{13}$ should occur infrequently in revaccinated individuals. The study also suggests ways to determine correlates of protection in the absence of true smallpox challenge.

While the study by Hammarlund et al. indicates that most vaccinated individuals may retain some immunity against smallpox infection, Weltzin et al. introduce a first-generation replacement for those without immunity. This is an important accomplishment because the current vaccine stocks, even if diluted, would probably not fulfill the demands of unvaccinated individuals in the United States, let alone worldwide ${ }^{5,8,9}$.

Weltzin et al. adapted the existing Dryvax vaccine, which is derived from the crossprotective vaccinia virus, to a human cell line for production in tissue culture. After animal studies, they chose one clone as the most promising vaccine candidate. In a small clinical study in humans, $100 \%$ subjects vaccinated with the new vaccine (ACAM-100) vs. $97 \%$ of Dryvax-vaccinated subjects exhibited the hallmark of vaccine take, a significant cutaneous reaction at the site of scarification. Overall, ACAM-100 induced a robust immune response, although there were differences between the two vaccines. For example Dryvax induced higher antibody titers while ACAM-100 vaccination seemed to result in stronger CD4 $\mathrm{T}$ cell responses. The vaccines had similar safety profiles, with each participant experiencing at least one mild to moderate adverse event.
Unfortunately, Weltzin et al. did not use the same assays as those used in the longitudinal studies of Hammarlund et al. In the future, such comparisons would increase confidence in this new vaccine. Challenge studies in nonhuman primate models are also warranted.

The safety profile of ACAM1000 seems similar to, and perhaps slightly better than, that of Dryvax. However, the investigators still observed a low rate of lethality in suckling mouse studies, as well as the induction of encephalitis in nonhuman primates, as is the case with Dryvax. In addition, the issue of cardiac toxicity, which has recently plagued vaccination efforts, needs to be clinically evaluated $^{14}$. In fact, the mass vaccination of health-care workers and law-enforcement personnel has recently been put on hold because of safety issues.

Until proven safe, only when faced with the threat of a smallpox outbreak should such a vaccine be considered for mass vaccination campaigns. The ACAM1000 vaccine, rather than being a truly new approach, is a modern production strategy for an old vaccine. The goal of a new safe and effective smallpox vaccine has not yet been attained ${ }^{15,16}$, but a suitable stopgap seems to be in place.

These two studies provide new technology as well as a suitable benchmark for the development of the next generation of vaccines. However, the reagents necessary for detailed and defined analyses of humoral and cellular responses in this effort are still lacking. A central repository for reagents to study agents of bioterrorism will significantly aid this effort. In addition, pharmaceutical approaches that could be useful in treating smallpox infection should be moved forward to testing in nonhuman primate studies as rapidly as possible. Such therapeutic agents will further alleviate vaccine dependence and provide an additional cushion of safety.

1. Alibek, K. Biohazard (ed. Handelman, S.) 107-122 (Delta Publishing, New York, 1999).

2. Preston, R. The Demon in the Freezer 1-49 (Random House, New York, 2002).

3. Gellman, B. 4 nations thought to possess smallpox. Iraq, N. Korea named, two officials say. Washington Post November 5 (2002), p.A1.

4. Henderson, D.A. The looming threat of bioterrorism. Science 283, 1279-1282 (1999).

5. LeDuc, J.W. \& Becher, R. Current status of smallpox vaccine. Emerg. Infect. Dis. 5, 593-594 (1999).

6. Hammarlund, E. et al. Duration of antiviral immunity after smallpox vaccination. Nat. Med. 9, 1131-1137 (2003).

7. Weltzin, R. et al. Clonal vaccinia virus grown in cell culture as a new smallpox vaccine. Nat. Med. 9, 1125-1130 (2003)

8. Kaplan, E.H, Craft, D.L. \& Wein, L.M. Emergency response to smallpox attack: the case for mass vaccination. Proc. Natl. Acad. Sci. USA 99, 10935-10940 (2002)

9. Rosenthal, S.R., Merchlinsky, M., Kleppinger, C. \& Goldenthal, K.L. Developing new smallpox vaccines. Emerg. Infect. Dis. 7, 1-12 (2001).

10. Mack, T.M., Nobel, J.R. \& Thomas, D.B. A prospective study of serum antibody and protection against smallpox. Am. J. Trop. Hyg. 21, 214-218 (1972).

11. Frwy, S.E., Newman, F.K., Yan, L. \& Belshe, R.B. Responses to smallpox vaccine in persons immunized in the distant past. JAMA 289, 3295-3299 (2003).

12. McConkey, S.J. et al. Enhanced T-cell immunogenicity of plasmid DNA vaccines boosted by recombinant modified vaccinia virus Ankara in humans. Nat. Med. 9, 729-735 (2003)

13. Copeman, P.W.M. \& Wallace, H.J. Eczema vaccinatum. Br. Med. J. 2, 906-908 (1964).

14. Cangemi, V.F. Acute pericarditis after smallpox vaccination. N. Engl. J. Med. 258, 1257 (1958).

15. Neff, J.M. et al. Complications of smallpox vaccination. I. National survey in the United States, 1963. N. Engl. J. Med. 276, 125-132 (1967).

16. Lane, J.M., Ruben, F.L., Abrutyn, E. \& Millar, J.D. Deaths attributable to smallpox vaccination, 1959 to 1966, and 1968. JAMA 212, 441-444 (1970).

\title{
Counteracting calcification
}

Despite high levels of phosphate and calcium in the body, mineralization normally occurs only in bones and teeth. But when calcification afflicts other regions of the body, as occurs in diseases such as atherosclerosis and chronic renal failure, tissues can be severely damaged. What keeps these minerals in check? In the 1 August Journal of Cell Biology Schäfer et al. provide evidence that an abundant serum protein negatively regulates calcification. The investigators examined mice deficient in this protein, a cysteine protease named $\alpha_{2}$-Heremans-Schmid glycoprotein (Ahsg, also known as fentuin-A). The mice were living on the edge. They appeared phenotypically normal but developed severe calcification of various organs while on a mineral- and vitamin D-rich diet. Shown here are calcified deposits in small vessels in muscles along vertebrae, from an Ahsg-deficient mouse fed the enriched diet for 4 months (inset, from wild-type mouse). The Ahsg-deficient mice, in combination with a genetic background sensitized to calcification, also developed severe calcification. Moreover, patients with calciphylaxis. a severe calcification disorder, had low serum Ahsg. The results jibe with previous in vitro studies showing that Ahsg inhibits formation and precipitation of basic calcium phosphate, a mineral central to bone formation.

Charlotte Schubert

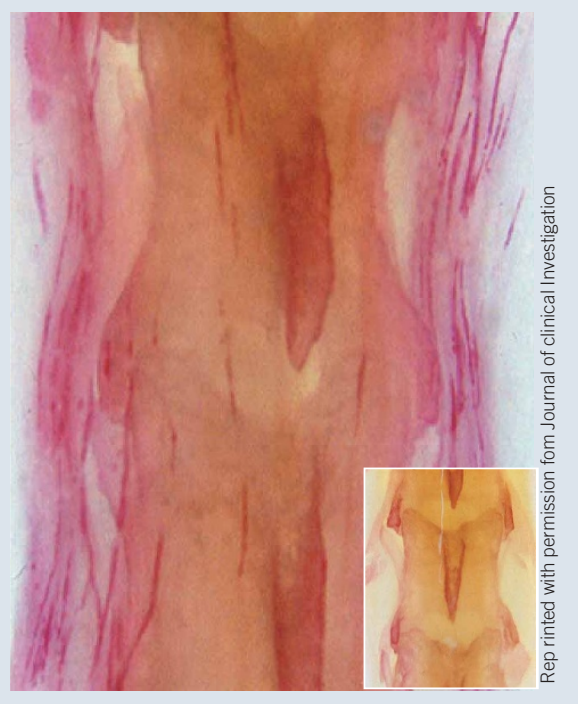

